\title{
Multiplex Raman imaging of organelles in endothelial cells
}

\author{
Ewelina Matuszyk ${ }^{1 *}$, Adriana Adamczyk ${ }^{1,2}$, Basseem Radwan ${ }^{1,2}$, Anna Pieczara ${ }^{1}$, Piotr \\ Szcześniak $^{3}$, Jacek Mlynarski ${ }^{3}$, Katarzyna Kamińska ${ }^{2}$, Malgorzata Baranska ${ }^{1,2 *}$ \\ ${ }^{1}$ Jagiellonian Centre for Experimental Therapeutics (JCET), Jagiellonian University, 14 \\ Bobrzynskiego Str., 30-348 Krakow, Poland \\ ${ }^{2}$ Faculty of Chemistry, Jagiellonian University, 2 Gronostajowa Str., 30-387 Krakow, Poland \\ ${ }^{3}$ Institute of Organic Chemistry, Polish Academy of Sciences, 44/52 Kasprzaka Str., 01-224 \\ Warsaw, Poland
}

*Correspondence: m.baranska@uj.edu.pl \& ewelina.matuszyk@uj.edu.pl

Keywords: Raman microscopy, molecular Raman reporters, tags, cells, nucleus, lipids, mitochondria.

\begin{abstract}
Raman imaging using molecular reporters is a relatively new approach in subcellular investigations. It enables the visualization of organelles in cells with better selectivity and sensitivity compared to the label-free approach. Essentially Raman reporters possess in their structure an alkyne molecular group that can be selectively identified in the spectral region silent for biomolecules, hence facilitate the localization of individual organelles. The aim of this work is to visualize the main cell organelles in endothelial cells (HMEC-1) using established reporters (EdU and MitoBADY), but also to test a new one, namely falcarinol, which exhibits lipophilic properties. Moreover, we tested the possibility to use Raman reporters as a probe to detect changes in distribution of certain organelles after induced endothelial dysfunction (ED) in in vitro models. In both cases, induced ED is characterized by the formation of lipid droplets in the cells, which is why a good tool for the detection of lipid-rich organelles is so important in these studies. Twodimensional Raman images were obtained, visualizing the distribution of selected organic compounds in the cell, such as proteins, lipids, and nucleic acids. Additionally, the distribution of EdU, MitoBADY and falcarinol in endothelial cells was determined. Moreover, we highlight some drawback of established Raman reporter and the need for testing them in various physiological state of the cell.
\end{abstract}




\section{Introduction}

Label-free Raman imaging is a well- established method in biomedical research with a potential of application for medical diagnostics in the future [1]. However, beside this straightforward use of Raman microscopy a new approach based on molecular reporters seems to be very promising an constantly evolving [2,3]. These chemical compounds introduced to cells, in micromolar concentrations, targeting specific organelles allow for their visualization, relatively faster and better than it is done in a label-free manner. Raman reporters usually show characteristic band in region spectrally silent for biological samples $\left(1800-2800 \mathrm{~cm}^{-1}\right)$, hence the spectra collected from a specific cell area show precisely where the reporter accumulates, and thus, enable the localisation of individual organelles with better sensitivity and selectivity [2-5].

One of the reporters is alkyne-tagged thymidine analogue 5-ethynyl-2'-deoxyuridine (EdU), which incorporates to newly synthesized DNA double-strand. It has been widely used for copper(I) catalysed azide [3+2] cycloadditions with a fluorescent probe in what is called 'click chemistry' to study cell proliferation [6]. EdU has been used to visualize nuclei of human cervical cancer cell line (HeLa) by detecting its ethynyl group vibration at $2120 \mathrm{~cm}^{-1}$ [3]. While EdU binds specifically to cellular DNA, ethynyluridine (EU) interacts with RNA that allows for detection of the two nucleic acids independently, what is usually not possible using label-free Raman imaging [7]. Visualization of mitochondria of HeLa cells was possible after utilizing bisarylbutadiyne (BADY) combined with lipophilic triphenylphosphonium cation (TPP+) as a mitochondrial targeting moiety. The signal from the developed tag (MitoBADY) co-localizing with cytochrome $c$ shows the potential of MitoBADY to be used as a Raman reporter for mitochondria [2]. Similarly, Am-CN Azo-OH was conjugated to TPP+ (Mito-Azo) and used as a resonance Raman probe for mitochondria [8]. Moreover, polyene-based reporters coupled with targeting moieties have been used to image specific subcellular structures including mitochondria [5]. Although Raman imaging of lipids is relatively easy due to their large cross-section for Raman scattering, different molecular reporters for lipids have been also developed. The alkyne labelled lipid precursor, 17-octadecynoic acid (17-ODYA), has been used to track lipid distribution in cells simultaneously with other Raman tags [7]. Furthermore, polyene-based (Carbow2202) has been designed to track cellular lipid droplets (LDs) [5]. In our previous work, we have demonstrated the potential of astaxanthin as a resonance Raman probe for lipids in endothelial cells (ECs) [9].

Vascular endothelium plays a vital role in maintaining cardiovascular homeostasis acting as a unique organ with multiple endocrine, paracrine and autocrine functions. Endothelial dysfunction (ED) has been associated with various diseases such as hypertension, diabetes and atherosclerosis, where ED could be either the cause or consequence of such events [10]. In many cases, alteration in the function of endothelial cell organelles could lead to ED. For instance, endothelial inflammation is linked to increased expression of pro-inflammatory cytokines and activation of transcription factors. Vascular calcification has been suggested as a consequence of DNA damage response suppression due to chromatin compression [11]. In turn, mitochondrial overproduction of Reactive Oxygen Species has been shown to inhibit endothelial nitric oxide synthase eNOS activity in association with hyperglycemia and atherosclerosis [12,13]. 
Furthermore, abnormal lipid accumulation leads to lipotoxicity causing oxidative stress, and the alteration of LDs distribution and composition has been recognised as a marker for endothelial inflammation $[14,15]$. Therefore, following the changes in ECs on the subcellular level is crucial for understanding the development of ED.

ED can be studied in vitro in various models of development of specific disease. Here we used two models, where cells were treated with the tumour necrosis factor alpha (TNF- $\alpha$ ) and chloroquine drug, respectively. TNF- $\alpha$, a pro-inflammatory cytokine, is widely applied to model cell inflammation in vitro. Stimulation of ECs with TNF- $\alpha$ results in several biochemical changes of which the most distinctive for Raman spectroscopy measurements is the alteration of lipids composition and distribution, specifically observed as a higher number of LDs of unsaturated character $[14,15]$. On the other hand, chloroquine, in therapeutic doses, works as an antimalarial and anti-inflammatory drug. Moreover, chloroquine induces phospholipidosis - formation of phospholipid- and cholesterol-rich bodies in the cytoplasm area [16,17]. Importantly, lipid-related changes in intracellular milieu can be straightforwardly investigated with Raman spectroscopy. In higher concentrations, chloroquine can increase NO synthesis in ECs by impairment of iron metabolism at intracellular acid compartments [18].

Previous studies have utilized Raman reporters for better and faster visualization of subcellular structures; thus, we believe that labelled Raman imaging could have also a potential in studding changes occurring at subcellular level in models of ED.

The aim of this work is to utilize Raman molecular reporters to visualize the main cellular organelles, i.e. nucleus, mitochondria, and perinuclear area, where biochemical changes occur together with development of ED. As a proof-of-concept two different models of ED are used, i.e. cells are stimulated with TNF- $\alpha$ and chloroquine, respectively. Another focus of this work is a new reporter to be tested for lipids, falcarinol, an anti-inflammatory polyacetylene occurring naturally in the plants of Apiaceae family. ED is often manifested by changes in accumulation and composition of lipids in ECs, therefore, developing new reporters for fast detection of cellular lipids is of interest.

\section{Material and methods}

\subsection{Cells}

A HMEC-1 cell line of human dermal microvascular endothelial cells was chosen to investigate subcellular distribution of EdU, MitoBADY and falcarinol. The cells for Raman measurements were directly seeded onto uncoated $\mathrm{CaF}_{2}$ slides $(25 \times 2 \mathrm{~mm}$, Crystran LTD) at a concentration of $1.2 \cdot 10^{5}$ cells per well to achieve the confluence at the level of about $70 \%$. Cells were cultured in complete MCDB131 medium (Gibco Life Technologies) supplemented with $10 \mathrm{mM}$ L-glutamine (Gibco Life Technologies), $1 \mu \mathrm{g} \cdot \mathrm{mL}^{-1}$ hydrocortisone (Sigma Aldrich), $10 \mathrm{mg} \cdot \mathrm{mL}^{-1}$ epidermal growth factor (EGF, Sigma Aldrich), 10\% fetal bovine serum (FBS, Gibco Life Technologies) and antibiotic antimycotic solution (AAS with $10.000 \mathrm{U}$ penicillin, $10 \mathrm{mg}$ streptomycin and $25 \mu \mathrm{g}$ amphotericin B per $\mathrm{mL}$ ) and maintained at $37^{\circ} \mathrm{C}$ in atmosphere of air with $5 \% \mathrm{CO}_{2}$ in a cell culture 
incubator. After 24 hours of incubation, enabling the attachment of all cells to the slide surface, they were rinsed twice with phosphate buffered saline (PBS, pH 7.4, Gibco Life Technologies) and exposed to different experimental conditions. Firstly, the HMEC-1 cells were incubated separately with EdU (20 $\mu \mathrm{M}, 24 \mathrm{~h}$; Abcam), MitoBADY (400 nM, $2 \mathrm{~h}$; synthesized according to protocol described elsewhere [2]) and falcarinol (50 $\mu \mathrm{M}, 2 \mathrm{~h}$; Cayman chemicals) as presented in Figure 3. The distribution of falcarinol was tested after pretreatment with chloroquine $(10 \mu \mathrm{M}, 24 \mathrm{~h}$; Sigma Aldrich) or TNF- $\alpha$ (10 ng/ml, $24 \mathrm{~h}$; Sigma Aldrich), as presented in Figure 2. The same pretreatment was used prior the incubation with MitoBADY, as presented in Figure 4. Untreated HMEC-1 cells maintained in the medium were used as a control. After treatment of the cells described above, samples were washed twice with PBS and fixed with $0.5 \%$ solution of glutaraldehyde in PBS for 4 minutes. The samples were stored in the PBS buffer at $4{ }^{\circ} \mathrm{C}$ prior to Raman measurements. The experiments were performed in two biological repetitions.

\subsection{Raman imaging}

Raman imaging of all samples was performed using a WITec alpha 300 Confocal Raman Imaging system (WITec $\mathrm{GmbH}$ ) equipped with a UHTS 300 spectrograph, a CCD detector (Andor, DU401A-BV-352), a 60× water-immersive objective (Nikon Fluor, NA = 1.0) and a 20× air objective (Zeiss EC Epiplan, NA=0.4). The air-cooled solid-state laser with the excitation wavelength of $532 \mathrm{~nm}$ was used to excite the sample. The spectra were recorded with the spectral resolution of $3 \mathrm{~cm}^{-1}$. Cells were measured with $0.5 \mathrm{~s}$ integration time, the solutions of investigated Raman reporters were measured with $1 \mathrm{~s}$ integration time and 25 accumulations. The laser power at the sample was $20 \mathrm{~mW}$. For each biological repetitions of experiment, 5-7 cells were measured.

\subsection{Data Analysis}

Raman data analysis was performed using a WITec Plus software (WITec GmbH). The Raman data was analyzed with $k$-Means Cluster Analysis (KMCA) using the Manhattan distance algorithm. Cluster analysis was carried out after cosmic spike removal and background subtraction (polynomial fit, order=3). Raman distribution images were obtained based on the integration of respective marker bands. Averaged spectra were obtained by averaging Raman spectra from analyzed area of n-number of cells. All presented spectra were vector normalized in the range of $3100-600 \mathrm{~cm}^{-1}$. For data presentation Origin(Pro) 2020 (OriginLab Corporation) was used.

\section{Results and discussion}

\subsection{Spectroscopic characterization of Raman reporters}

Previously it has been demonstrated that Raman microscopy enables visualization of essential components of cells and identification of biochemical alterations in the ECs after their exposure to several factors. Moreover, Raman microscopy gives the possibility to determine the efficiency of cellular uptake of various compounds, e.g. drugs, fatty acids, or nanoparticles [19-23]. Both these advantages of Raman microscopy, i.e. high resolution and high sensitivity in detection of specific 
molecules, can be combined and applied for in vitro studies using molecular reporters, in order to image the subcellular organelles, i.e. mitochondria, nucleus or lysosomes $[2,8,24,25]$. Here, we tested a few reporters on HMEC-1 cell line, using them individually and multiplexed. Cells were exposed to the well-established tags, such as EdU, MitoBADY, as well as to the new one, naturally occurring polyacetylene, namely falcarinol.

Figure 1A shows the chemical structure and respective Raman spectrum of EdU. An alkyne band used for its identification is positioned at $2120 \mathrm{~cm}^{-1}$. MitoBADY is a mitochondria-targeting Raman probe based on alkynyl structure, bisarylbutadiynes (BADY) connected with targeting moiety, triphenylphosphonium $\left(\mathrm{TTP}^{+}\right)$that accumulates in the inner mitochondrial matrix $[2,26]$. Its chemical structure and Raman spectrum is displayed at Figure 1B. An intense alkyne band, much stronger than in the case of EdU, can be seen at $2220 \mathrm{~cm}^{-1}$, and some other less intense bands are observed at $1603 \mathrm{~cm}^{-1}, 1345 \mathrm{~cm}^{-1}$ and $1187 \mathrm{~cm}^{-1}$. Subsequently, the structure and respective Raman spectrum of last tested compound, namely falcarinol is presented at Figure 1C. An intense alkyne band is observed at $2258 \mathrm{~cm}^{-1}$ due to conjugated $-\mathrm{C} \equiv \mathrm{C}$ - triple bond of the falcarinol chain [27].

A

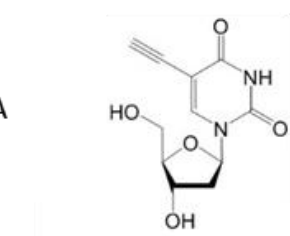

B
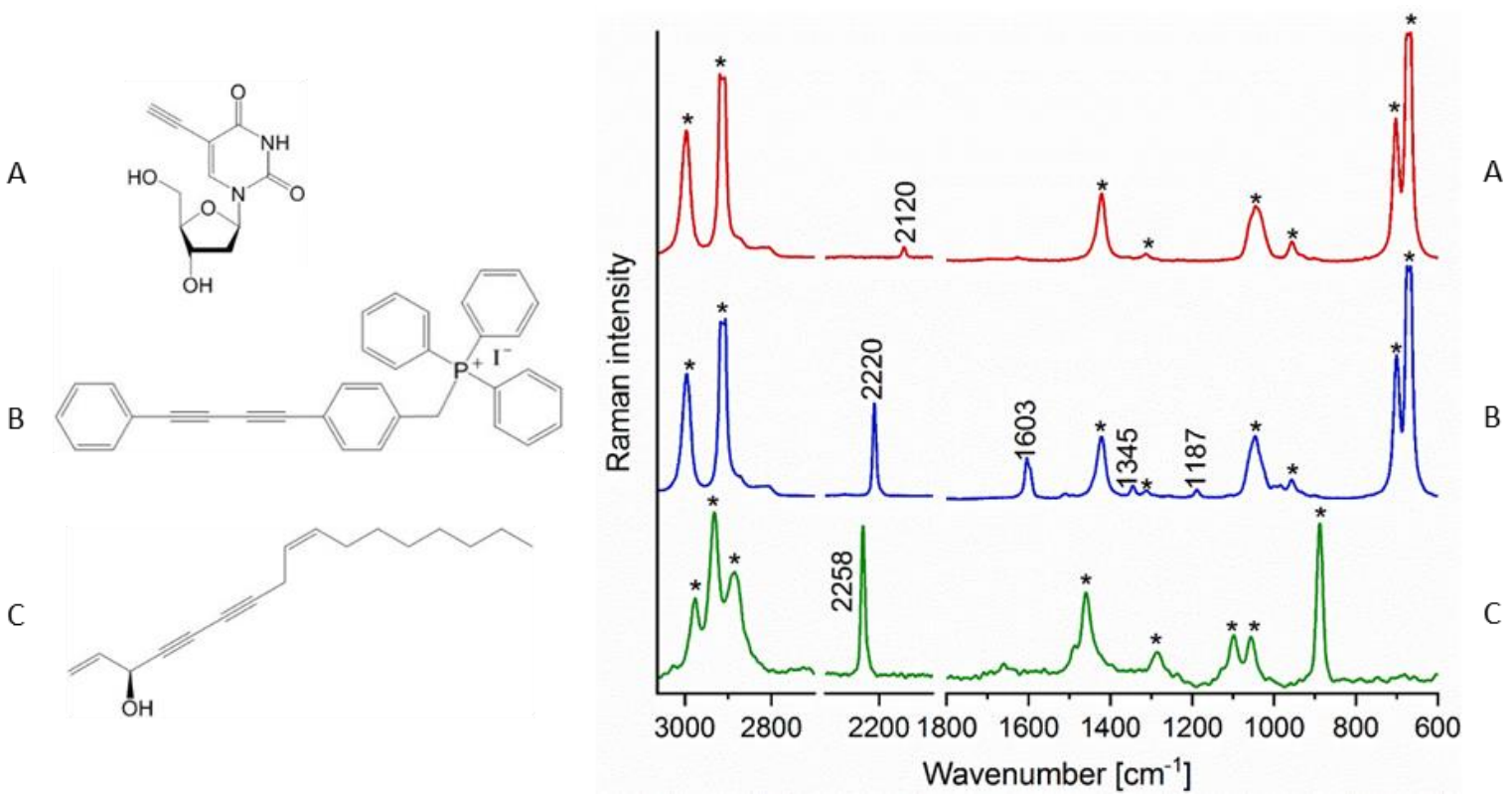

Figure 1. Chemical structure of Raman spectra of (A) EdU, (B) MitoBADY, (C) falcarinol together with respective Raman spectra of studied reporter as a $10 \mathrm{~g} / \mathrm{L}$ solution in DMSO (A, B) and ethanol (C). Bands derived from the solvents are marked by stars.

\subsection{Falcarinol as a new reporter to visualize lipids in models of endothelial dysfunction}

Most Raman reporters are designed in a way to give a signal in the range that is spectrally silent for biological samples, e.g. that relays on the presence of $-\mathrm{C} \equiv \mathrm{C}$ - group in the molecular structure. Therefore, we decided to evaluate the utility of naturally occurring polyacetylene falcarinol with two carbon-carbon triple bonds to be considered as a candidate for Raman reporter 
itself or after functionalization with targeting group. However, there is a gap in a knowledge about subcellular localization of falcarinol after incubation with mammalian cells. Due to lipophilic properties it is expected to accumulate in lipidic structures e.g. ER or LDs. In order, to investigate subcellular distribution of falcarinol, we used two agents that induces lipid-related changes in endothelial cells, namely TNF- $\alpha$ and chloroquine pretreatment. As can be seen in Figure 2, Raman images obtained by the integration of band in a range of $2900-2830 \mathrm{~cm}^{-1}$ (lipids) and $2270-2250$ $\mathrm{cm}^{-1}$ (falcarinol) show comparable localization. Interestingly, presence of falcarinol does not entirely exhibit the pattern of LDs distribution. Its stronger affinity to ER located in perinuclear area requires however further investigations.

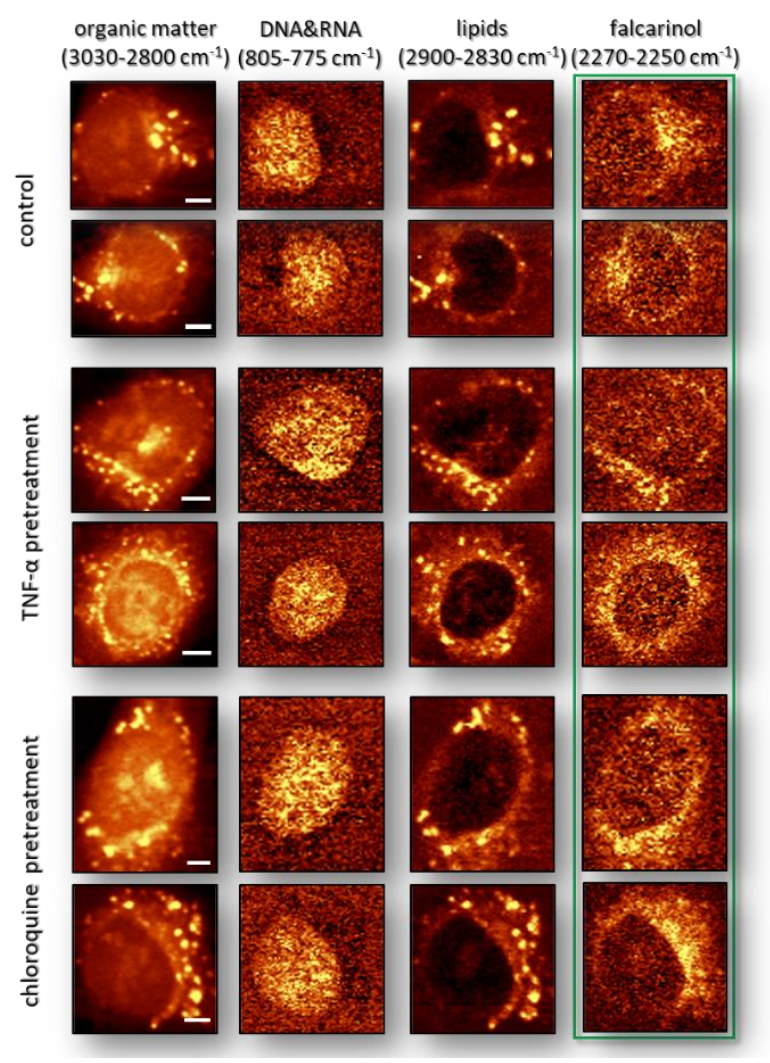

Figure 2. Representative Raman images of HMEC-1 cells incubated with falcarinol ( $400 \mathrm{nM}, 2$ h): control, and cells preincubated with TNF- $\alpha$ and with chloroquine, respectively. The images were obtained by the integration of Raman bands over the selected spectral regions: $3030-2830 \mathrm{~cm}^{-1}$ (organic matter), $2900-2830 \mathrm{~cm}^{-1}$ (lipids), $805-775 \mathrm{~cm}^{-}$ ${ }^{1}$ (DNA\&RNA) and $2270-2250 \mathrm{~cm}^{-1}$ (falcarinol), respectively.

\subsection{Intracellular distribution of EdU, MitoBADY and falcarinol in endothelial cells}

In this section results of labeling HMEC-1 cells with EdU, MitoBADY and falcarinol are presented, respectively. The aim was to evaluate the effectiveness of chosen Raman reporters in visualization of different compartments of ECs. For this purpose, two-dimensional Raman images based on characteristic bands of selected organic compounds such as proteins, lipids, and nucleic acids (DNA and RNA) show their distribution in ECs (Figure 3). The recorded Raman spectra were 
analyzed by integration of the intensity of characteristic bands assigned to the $\mathrm{C}-\mathrm{H}$ stretching vibrations (in the region of $3030-2830 \mathrm{~cm}^{-1}$ ), the ring breathing modes in the DNA/RNA bases (in the region of $805-775 \mathrm{~cm}^{-1}$, the $\mathrm{C}-\mathrm{H}$ stretching vibrations originating mostly from lipids (in the region of $2900-2830 \mathrm{~cm}^{-1}$ ) [28]. Additionally, distribution of EdU, MitoBADY and falcarinol was demonstrated based on symmetric stretching of $-\mathrm{C} \equiv \mathrm{C}$ - in EdU (in the range of $2136-2106$ $\mathrm{cm}^{-1}$ ), MitoBADY (in the range of $2236-2206 \mathrm{~cm}^{-1}$ ) and falcarinol (in the range of $2270-2250$ $\mathrm{cm}^{-1}$ ), presented in Figure 3A, 3B and 3C, respectively. Figure 3D displays average Raman spectra taken from subcellular regions targeted by given Raman reporter, namely nucleus area (where EdU has located), cytoplasm area where MitoBADY and falcarinol were observed. Measurement protocols chosen here resulted in comparable intensity of the alkyne bands for each Raman reporter.
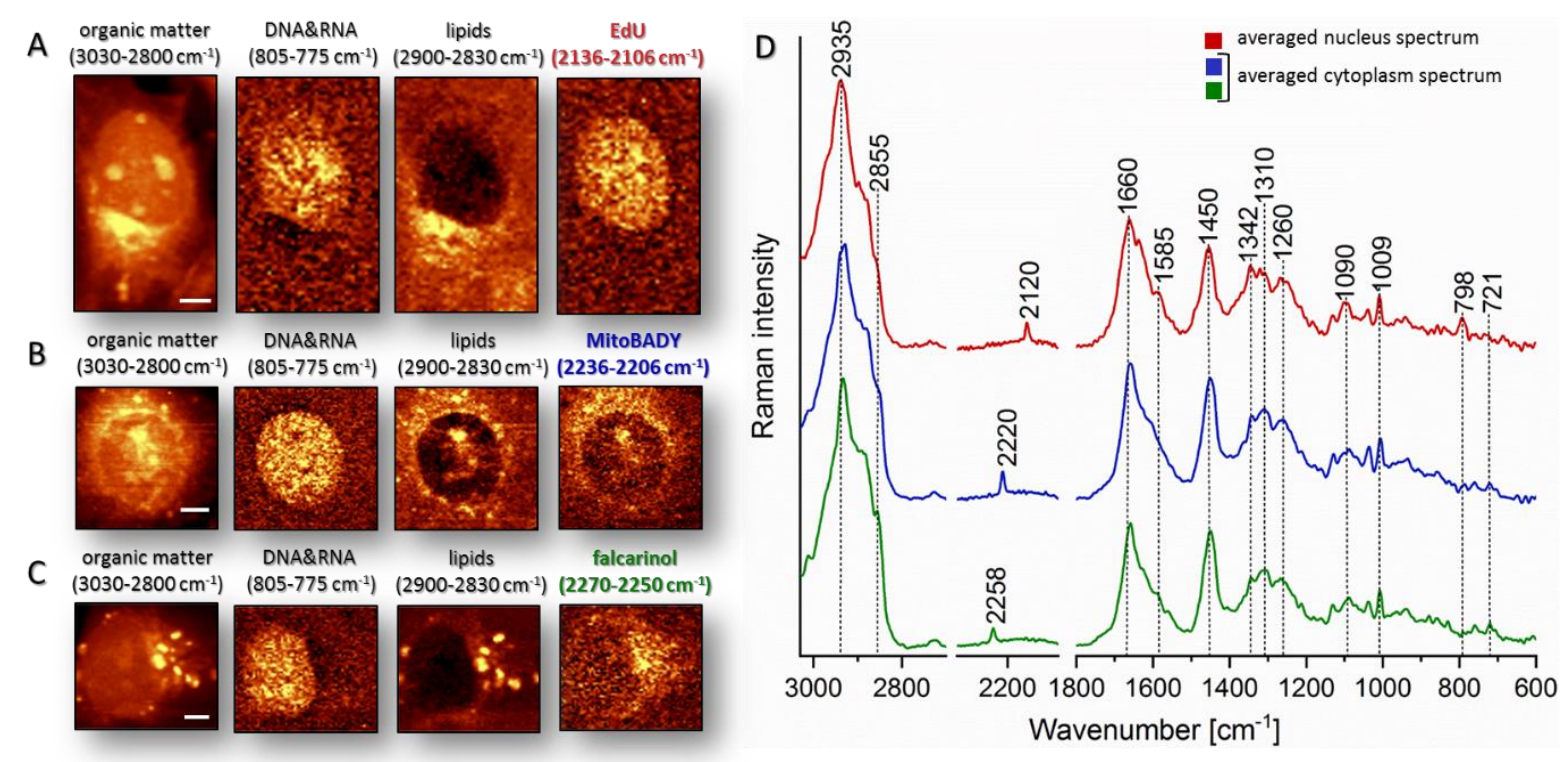

Figure 3. Representative Raman images of HMEC-1 cells incubated with (A) EdU (20 $\mu \mathrm{M}, 24 \mathrm{~h}$ ), (B) MitoBADY $(400 \mathrm{nM}, 2 \mathrm{~h})$ and $(\mathrm{C})$ falcarinol $(50 \mu \mathrm{M}, 2 \mathrm{~h})$ obtained by the integration of Raman bands over the selected spectral regions: $3030-2830 \mathrm{~cm}^{-1}$ (organic matter), $2900-2830 \mathrm{~cm}^{-1}$ (lipids), $805-775 \mathrm{~cm}^{-1}$ (DNA\&RNA) and $2136-2106$ $\mathrm{cm}^{-1}$ (EdU), $2236-2206 \mathrm{~cm}^{-1}$ (MitoBADY), $2270-2250 \mathrm{~cm}^{-1}$ (falcarinol), respectively along with (D) the averaged spectra of nucleus of cell incubated with EdU (red), and cytoplasm of cells incubated with MitoBADY (blue) and falcarinol (green). Scale bars equal $5 \mu \mathrm{m}$.

As can be seen in Figure 3A, incubation of HMEC-1 with EdU allowed for visualization of nucleus area with better contrast in comparison to image obtained by integration of Raman band originating from endogenous nucleic acids $\left(789 \mathrm{~cm}^{-1}\right)$. On the other hand, imaging of mitochondria in non-label way is based on the resonant Raman bands of cytochrome $c$, an endogenous heme protein associated with the inner mitochondrial membrane [29]. However, detection of cytochrome $c$ depends strongly on cell types, physiological state of the cell and this approach, in general, applies to live cell imaging [30]. Therefore, introduction of exogenous molecules (Raman reporters) that accumulate specifically in mitochondria was proposed as an alternative, more universal, method 
[2,5,8,31]. As mentioned in the introduction, MitoBADY has been previously tested on HeLa cells and its subcellular distribution corresponds well with that of cytochrome $c$ [2]. Although, mitochondrial content in ECs is modest compared to other cell types, as they occupy 2-6\% of cytoplasmic volume, one of the ED mechanism is solely related to disruption of mitochondrial functioning [13,32]. Moreover, mitochondrial distribution within the cell has been predicted to influence mitochondrial signaling in the endothelium [13,33]. Therefore, a new method for detection of this organelle would be beneficial. Here, we synthesized and tested MitoBADY using HMEC-1 cell line. Figure 3B displays images of HMEC-1 cells incubated with MitoBADY for 2 $\mathrm{h}$ at $400 \mathrm{nM}$ concentration. Due to relatively small number of mitochondria, the incubation time was extended compared to original protocol [2], in order to obtain reasonable intensity of the band at $2220 \mathrm{~cm}^{-1}$. The spatial distribution of MitoBADY was observed in the cytoplasm area. HMEC1 cells were incubated with falcarinol for $2 \mathrm{~h}$ at the concentration of $50 \mu \mathrm{M}$, and imaged, as presented at Figure 3c. The images indicate that falcarinol accumulates in the lipidic area of the cell in close vicinity to nucleus, as mentioned in section 3.2.

ECs have ability to form LDs, especially after incubation with certain agents such as fatty acids [34] or TNF- $\alpha$ [14]. Our study led us to conclusion, that due to lipophilic properties, MitoBADY except accumulation in mitochondria can be also observed in other lipidic substructures e.g. LDs, that may provide misleading results. Figure 4A displays Raman images of HMEC-1 cells incubated with MitoBADY, i.e. control, and cells pretreated with TNF- $\alpha(10 \mathrm{ng} / \mathrm{ml})$ and chloroquine (10 $\mu \mathrm{M}$ ), respectively. Control cells possess a few small LDs with similar pattern as TNF- $\alpha$ pretreated cells, while cells stimulated with chloroquine exhibit a high number of LDs. For better visualization of the distribution of lipids and MitoBADY, the respective false-color Raman images were combined generating the composite image shown at Figure 4B. The substantial colocalization of MitoBADY and lipidic structures was observed (white area at the composite image). Additionally, Raman spectrum of lipids is displayed (Figure 3B) where strong signal from MitoBADY (2220 $\mathrm{cm}^{-1}$ ) is present. This experiment was performed in order to highlight possible drawbacks of designed Raman reporters, especially for types of cells rich in lipidic structures. 

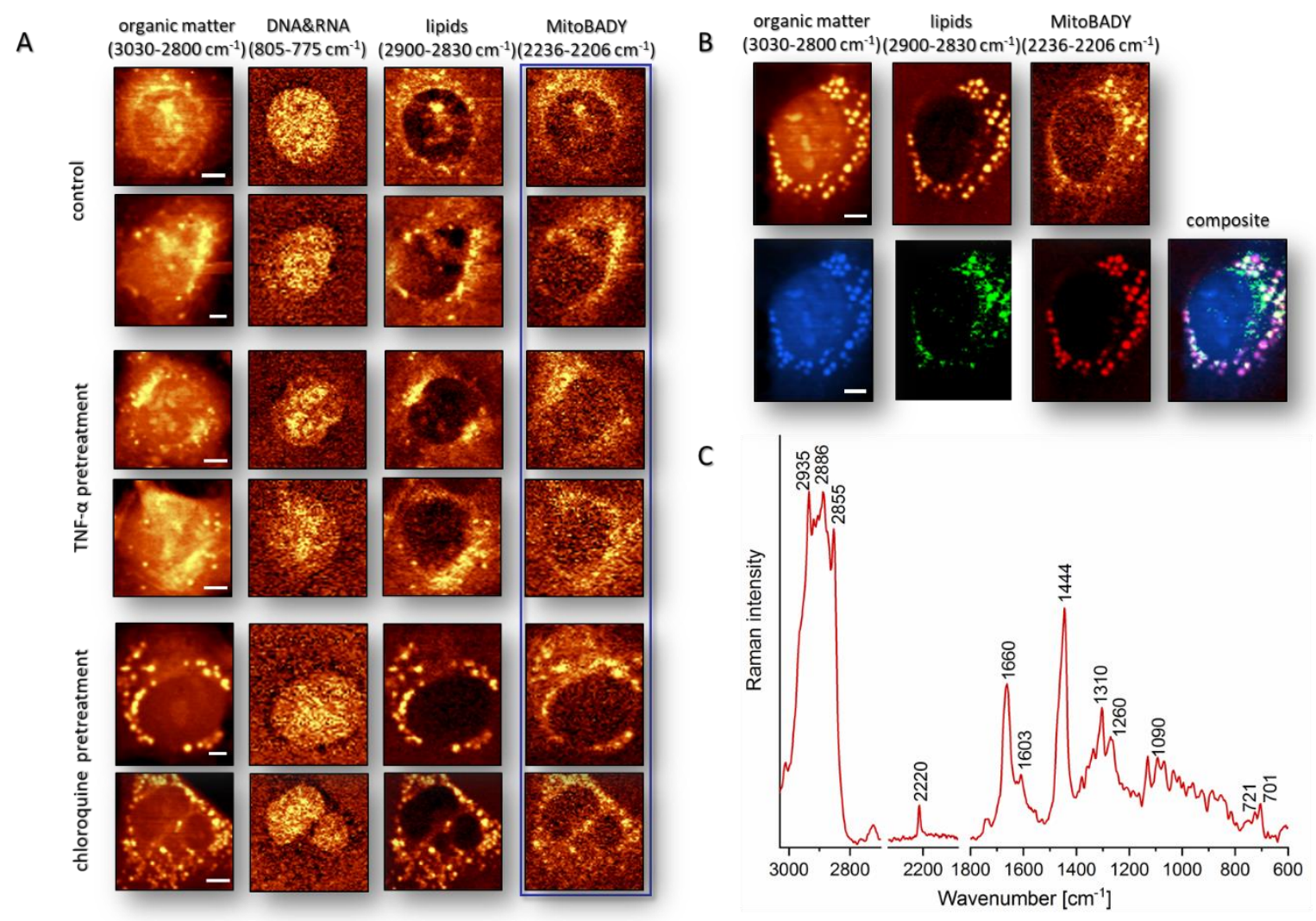

Figure 4. Representative Raman images of HMEC-1 cells incubated with (A) MitoBADY (400 nM, 2 h): control, and cells preincubated with TNF- $\alpha$ and with chloroquine, respectively. The images were obtained by the integration of Raman bands over the selected spectral regions: $3030-2830 \mathrm{~cm}^{-1}$ (organic matter), $2900-2830 \mathrm{~cm}^{-1}$ (lipids), 805 $775 \mathrm{~cm}^{-1}$ (DNA\&RNA) and $2236-2206 \mathrm{~cm}^{-1}$ (MitoBADY), respectively. Raman images of HMEC-1 cell incubated with MitoBADY and preincubated with chloroquine obtained by the integration of Raman bands over the selected spectral regions: $3030-2830 \mathrm{~cm}^{-1}$ (organic matter), $2900-2830 \mathrm{~cm}^{-1}$ (lipids), along with false color images displaying the distribution of selected cell components and their colocalization in the composite image (B) along with the averaged spectrum of LDs (C). Scale bars equal $5 \mu \mathrm{m}$.

\subsection{Multiplex labeling of endothelial cells using three Raman reporters}

Visualization of many distinct molecular species is crucial for understanding such complex systems as cells, especially if important biochemical processes take place in them. Typically, the method of choice for such purpose is the fluorescence microscopy. However, this technique suffers from limited number of resolvable colors, due to broad and featureless nature of fluorescence spectra. Spontaneous, as well as Stimulated Raman microscopy enables to overcome this limitation, due to narrow Raman bands [5,35]. As displayed at Figure 5A, we performed the multiplex labeling of HMEC-1, thanks to different Raman shift of alkyne band observed for EdU, MitoBADY and falcarinol shown in spectra at Figure 5B. The experimental conditions remained the same as those used for labeling cells with single reporter. Distribution of EdU allows for visualization of nucleus area, and MitoBADY was observed almost uniformly in the cytoplasm, 
while falcarinol is present in perinuclear area, what is in agreement with the results presented above. However here, all three 'staining' were performed simultaneously. It should be emphasized that the methodology described here does not limit the use of more molecular reporters for the simultaneous detection of multiple organelles in a cell.

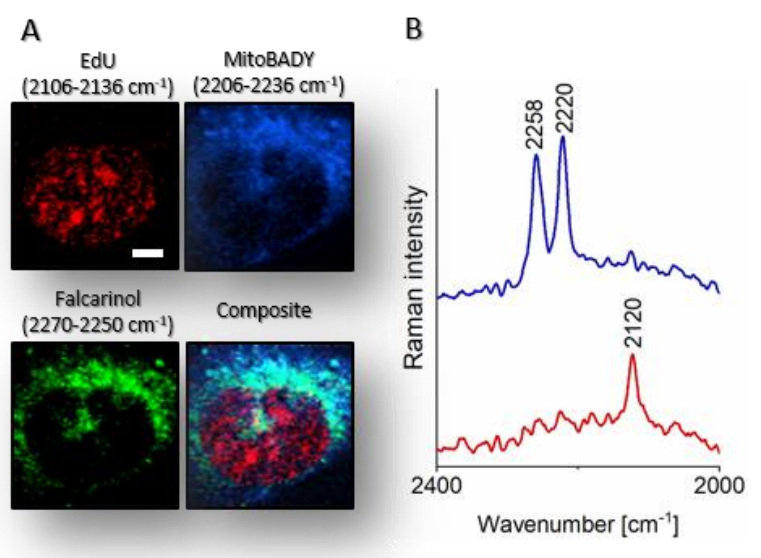

Figure 2. Representative false color Raman images of HMEC-1 cell incubated with EdU, MitoBADY and falcarinol (A) obtained by the integration of Raman bands over the selected spectral regions: $2136-2106 \mathrm{~cm}^{-1}$ (EdU), 2236 $2206 \mathrm{~cm}^{-1}$ (MitoBADY), $2270-2250 \mathrm{~cm}^{-1}$ (falcarinol) and combine image (composite) along with (B) the averaged spectra of cytoplasm (red) and nucleus (grey) with visible alkyne bands originating from falcarinol $\left(2258 \mathrm{~cm}^{-1}\right)$, MitoBADY $\left(2258 \mathrm{~cm}^{-1}\right)$ and EdU $\left(2120 \mathrm{~cm}^{-1}\right)$. Scale bar equals $5 \mu \mathrm{m}$.

\section{Conclusions}

In our research, we proved that the tested Raman reporters are able to locate and visualize specific cell organelles in the ECs. The cellular localization of falcarinol in mammalian cells was investigated for the first time. Our study showed that, falcarinol is mainly localized in lipid-rich perinuclear region. Interestingly, the distribution of falcarinol is not limited to the location of LDs. Its greater affinity for the ER, which is in the perinuclear area, requires further research. To the best of our knowledge, Raman reporters (in combination with spontaneous or stimulated Raman imaging) have been tested mainly on control cells, only to visualize the distribution of selected organelles or certain molecules. In this work, we tested application of Raman reporters as a tool to study intracellular changes caused by induced endothelial dysfunction in in vitro conditions. Here, we verified some possibilities as well as challenges of the labelled Raman imaging approach, which is considered to be constantly evolving and opening up new opportunities. In the conclusion, we consider that designing new effective Raman reporters, tested in various conditions will be beneficial for investigations in a field of biomedical research.

\section{CRediT authorship contribution statement}

M. Baranska - conceptualization; M Baranska, E. Matuszyk - methodology, E. Matuszyk, A. Adamczyk, P. Szczesniak, J. Mlynarski - investigation; E. Matuszyk, A. Adamczyk, A. Pieczara 
- data curation; E. Matuszyk, B. Radwan, K. Kaminska, A. Pieczara - writing-original draft preparation; M. Baranska, E. Matuszyk - writing - review and editing. All authors have read and agreed to the published version of the manuscript.

\section{Declaration of competing interest}

The authors declare no conflict of interest.

\section{Acknowledgement}

The study was supported by the grant LogicLab ITN funded by the European Union's Horizon 2020 research and innovation program under the Marie Skłodowska-Curie [grant agreement No 813920]. J.M. and P.S. thank the Foundation for Polish Science for financial support under award TEAM/2017-4/38. Authors are grateful to MSc Renata Budzynska (JCET JU) for cell culturing and MSc Ewelina Bik for supplying chloroquine.

\section{References}

[1] M. Baranska, A. Kaczor, K. Malek, A. Jaworska, K. Majzner, E. Staniszewska-Slezak, M.Z. Pacia, G. Zajac, J. Dybas, E. Wiercigroch, Raman microscopy as a novel tool to detect endothelial dysfunction, Pharmacol. Reports. 67 (2015) 736-743. doi:http://dx.doi.org/10.1016/j.pharep.2015.03.015.

[2] H. Yamakoshi, A. Palonpon, K. Dodo, J. Ando, S. Kawata, K. Fujita, M. Sodeoka, A sensitive and specific Raman probe based on bisarylbutadiyne for live cell imaging of mitochondria, Bioorganic Med. Chem. Lett. 25 (2015) 664-667. doi:10.1016/j.bmcl.2014.11.080.

[3] H. Yamakoshi, K. Dodo, A. Palonpon, J. Ando, K. Fujita, S. Kawata, M. Sodeoka, Alkyne-Tag Raman Imaging for Visualization of Mobile Small Molecules in Live Cells, J. Am. Chem. Soc. 134 (2012) 20681-20689. doi:10.1021/ja308529n.

[4] Y. Shen, Z. Zhao, L. Zhang, L. Shi, S. Shahriar, R.B. Chan, G. Di Paolo, W. Min, Metabolic activity induces membrane phase separation in endoplasmic reticulum, Proc. Natl. Acad. Sci. 114 (2017) 13394-13399. doi:10.1073/pnas.1712555114.

[5] F. Hu, C. Zeng, R. Long, Y. Miao, L. Wei, Q. Xu, W. Min, Supermultiplexed optical imaging and barcoding with engineered polyynes, Nat. Methods. 15 (2018) 194-200. doi:10.1038/nmeth.4578.

[6] T. Ishizuka, H.S. Liu, K. Ito, Y. Xu, Fluorescence imaging of chromosomal DNA using click chemistry, Sci. Rep. 6 (2016) 33217. doi:10.1038/srep33217.

[7] Z. Chen, D.W. Paley, L. Wei, A.L. Weisman, R.A. Friesner, C. Nuckolls, W. Min, Multicolor Live-Cell Chemical Imaging by Isotopically Edited Alkyne Vibrational Palette, J. Am. Chem. Soc. 136 (2014) 8027-8033. doi:10.1021/ja502706q.

[8] Y. Li, J. Heo, C.K. Lim, A. Pliss, A. V. Kachynski, A.N. Kuzmin, S. Kim, P.N. Prasad, Organelle specific imaging in live cells and immuno-labeling using resonance Raman probe, Biomaterials. 53 (2015) 25-31. doi:10.1016/j.biomaterials.2015.02.056. 
[9] K. Czamara, A. Adamczyk, M. Stojak, B. Radwan, M. Baranska, Astaxanthin as a new probe for biosensing of specific subcellular lipidic structures - can we detect lipids in cells under resonance Raman conditions?, Cell. Mol. Life Sci. (2020).

[10] K.-H. Park, W.J. Park, Endothelial Dysfunction: Clinical Implications in Cardiovascular Disease and Therapeutic Approaches, J. Korean Med. Sci. 30 (2015) 1213-1225. doi:10.3346/jkms.2015.30.9.1213.

[11] D. Melinda, C.A. M., S.C. M., DNA Damage Response, Arterioscler. Thromb. Vasc. Biol. 0 (2020) ATVBAHA.120.313792. doi:10.1161/ATVBAHA.120.313792.

[12] X.L. Du, D. Edelstein, S. Dimmeler, Q. Ju, C. Sui, M. Brownlee, Hyperglycemia inhibits endothelial nitric oxide synthase activity by posttranslational modification at the Akt site, J. Clin. Invest. 108 (2001) 1341-1348. doi:10.1172/JCI11235.

[13] M.A. Kluge, J.L. Fetterman, J.A. Vita, Mitochondria and Endothelial Function, Circ. Res. 112 (2013) 1171-1188. doi:10.1161/CIRCRESAHA.111.300233.

[14] K. Czamara, K. Majzner, A. Selmi, M. Baranska, Y. Ozaki, A. Kaczor, Unsaturated lipid bodies as a hallmark of inflammation studied by Raman 2D and 3D microscopy, Sci. Rep. 7 (2017) 40889. doi:10.1038/srep40889.

[15] E. Szafraniec, E. Kus, A. Wislocka, B. Kukla, E. Sierka, V. Untereiner, G.D. Sockalingum, S. Chlopicki, M. Baranska, Raman spectroscopy-based insight into lipid droplets presence and contents in liver sinusoidal endothelial cells and hepatocytes, J. Biophotonics. (2019) e201800290. doi:10.1002/jbio.201800290.

[16] N. Zheng, X. Zhang, G.R. Rosania, Effect of Phospholipidosis on the Cellular Pharmacokinetics of Chloroquine, J. Pharmacol. Exp. Ther. 336 (2011) 661-671. doi:10.1124/jpet.110.175679.

[17] N. Anderson, J. Borlak, Drug-induced phospholipidosis, FEBS Lett. 580 (2006) 5533-5540. doi:10.1016/j.febslet.2006.08.061.

[18] D. Ghigo, E. Aldieri, R. Todde, C. Costamagna, G. Garbarino, G. Pescarmona, A. Bosia, Chloroquine stimulates nitric oxide synthesis in murine, porcine, and human endothelial cells., J. Clin. Invest. 102 (1998) 595-605. doi:10.1172/JCI1052.

[19] K. Majzner, T. Wojcik, E. Szafraniec, M. Lukawska, I. Oszczapowicz, S. Chlopicki, M. Baranska, Nuclear accumulation of anthracyclines in the endothelium studied by bimodal imaging: fluorescence and Raman microscopy, Analyst. 140 (2015) 2302-2310. http://xlink.rsc.org/?DOI=C4AN01882F.

[20] E. Matuszyk, E. Sierka, M. Rodewald, H. Bae, T. Meyer, E. Kus, S. Chlopicki, M. Schmitt, J. Popp, M. Baranska, Differential response of liver sinusoidal endothelial cells and hepatocytes to oleic and palmitic acid revealed by Raman and CARS imaging, Biochim. Biophys. Acta - Mol. Basis Dis. 1866 (2020) 165763. doi:https://doi.org/10.1016/j.bbadis.2020.165763.

[21] B. Kann, H.L. Offerhaus, M. Windbergs, C. Otto, Raman microscopy for cellular investigations From single cell imaging to drug carrier uptake visualization, Adv. Drug Deliv. Rev. 89 (2015) 7190. doi:10.1016/j.addr.2015.02.006.

[22] F. Hu, S.D. Brucks, T.H. Lambert, L.M. Campos, W. Min, Stimulated Raman scattering of polymer nanoparticles for multiplexed live-cell imaging, Chem. Commun. 53 (2017) 6187-6190. doi:10.1039/C7CC01860F.

[23] C. Matthäus, S. Schubert, M. Schmitt, C. Krafft, B. Dietzek, U.S. Schubert, J. Popp, Resonance 
Raman Spectral Imaging of Intracellular Uptake of $\beta$-Carotene Loaded Poly(D, L -lactide- co glycolide) Nanoparticles, ChemPhysChem. 14 (2013) 155-161. doi:10.1002/cphc.201200577.

[24] A.N. Kuzmin, A. Pliss, C.K. Lim, J. Heo, S. Kim, A. Rzhevskii, B. Gu, K.T. Yong, S. Wen, P.N. Prasad, Resonance Raman Probes for Organelle-Specific Labeling in Live Cells, Sci. Rep. 6 (2016) 1-9. doi:10.1038/srep28483.

[25] H. Yamakoshi, K. Dodo, M. Okada, J. Ando, A. Palonpon, K. Fujita, S. Kawata, M. Sodeoka, Imaging of EdU, an alkyne-tagged cell proliferation probe, by Raman microscopy, J. Am. Chem. Soc. 133 (2011) 6102-6105. doi:10.1021/ja108404p.

[26] J. Zielonka, J. Joseph, A. Sikora, M. Hardy, O. Ouari, J. Vasquez-Vivar, G. Cheng, M. Lopez, B. Kalyanaraman, Mitochondria-Targeted Triphenylphosphonium-Based Compounds: Syntheses, Mechanisms of Action, and Therapeutic and Diagnostic Applications, Chem. Rev. 117 (2017) 10043-10120. doi:10.1021/acs.chemrev.7b00042.

[27] M. Baranska, H. Schulz, Spatial tissue distribution of polyacetylenes in carrot root, Analyst. 130 (2005) 855. doi:10.1039/b500975h.

[28] Z. Movasaghi, S. Rehman, I.U. Rehman, Raman Spectroscopy of Biological Tissues, Appl. Spectrosc. Rev. 42 (2007) 493-541. doi:10.1080/05704920701551530.

[29] P.L. Yeagle, Membrane Proteins, in: Membr. Cells, Elsevier, 2016: pp. 219-268. doi:10.1016/B978-0-12-800047-2.00010-3.

[30] M. Okada, N.I. Smith, A.F. Palonpon, H. Endo, S. Kawata, M. Sodeoka, K. Fujita, Label-free Raman observation of cytochrome c dynamics during apoptosis, Proc. Natl. Acad. Sci. 109 (2012) 28-32. doi:10.1073/pnas.1107524108.

[31] X. Li, M. Jiang, J.W.Y. Lam, B.Z. Tang, J.Y. Qu, Mitochondrial Imaging with Combined Fluorescence and Stimulated Raman Scattering Microscopy Using a Probe of the AggregationInduced Emission Characteristic, J. Am. Chem. Soc. 139 (2017) 17022-17030. doi:10.1021/jacs.7b06273.

[32] W.H. Oldendorf, M.E. Cornford, W.J. Brown, The large apparent work capability of the bloodbrain barrier: A study of the mitochondrial content of capillary endothelial cells in brain and other tissues of the rat, Ann. Neurol. 1 (1977) 409-417. doi:10.1002/ana.410010502.

[33] J. Park, J. Lee, C. Choi, Mitochondrial Network Determines Intracellular ROS Dynamics and Sensitivity to Oxidative Stress through Switching Inter-Mitochondrial Messengers, PLoS One. 6 (2011) e23211. doi:10.1371/journal.pone.0023211.

[34] K. Majzner, S. Chlopicki, M. Baranska, Lipid droplets formation in human endothelial cells in response to polyunsaturated fatty acids and 1-methyl-nicotinamide (MNA); confocal Raman imaging and fluorescence microscopy studies., J. Biophotonics. 9 (2016) 396-405. doi:10.1002/jbio.201500134.

[35] J.R. Lakowicz, ed., Principles of Fluorescence Spectroscopy, Springer US, Boston, MA, 2006. doi:10.1007/978-0-387-46312-4. 